\title{
HIGHER ORDER ESTIMATES AND SMOOTHNESS OF NONLINEAR WAVE EQUATIONS
}

\author{
MICHAEL C. REED
}

ABSTRACT. A completely elementary proof is given for Segal's nonlinear semigroup theorem.

The purpose of this paper is to give a completely elementary proof of an important theorem of Irving Segal [6]. Segal's results give conditions on the nonlinear terms in wave equations so that unique local, global, and smooth solutions exist and these techniques have found wide and important applications in the study of nonlinear equations; for examples, see the work of Strauss [7] or Chadam [1], [2]. The theorems which we prove are not exactly those of Segal and the differences are discussed at the end of the paper. We restrict our exposition to the Hilbert space case since, due to the discontinuity of the free wave group on the $L^{p}$ energy spaces [4], this is the case which arises in applications.

Let $A$ be a selfadjoint operator with domain $D(A)$ on a Hilbert space $\mathcal{H}$ and let $J$ be a densely defined mapping on $H$. The problem is to give conditions on $J$ that guarantee the existence, uniqueness, and smoothness of a solution to the Cauchy problem

$$
\phi^{\prime}(t)=-i A \phi(t)+J(\phi(t)), \quad \phi(0)=\phi_{0} .
$$

Theorem 1 (Local existence, uniqueness, smoothness). Let $A$ be a selfadjoint operator on a Hilbert space $\mathcal{H}$ and $N$ a positive integer. Suppose that $J$ is a densely defined mapping on $\mathcal{H}$ so that $J: D\left(A^{j}\right) \rightarrow D\left(A^{j}\right)$, for $0<j \leq N$, and:

$$
\left\|A^{j} J(\phi)\right\| \leq C\left(\|\phi\|, \cdots,\left\|A^{j} \phi\right\|\right)\left\|A^{j} \phi\right\|
$$

$\left(\mathrm{H}_{j}^{L}\right)\left\|A^{j}(J(\phi)-J(\psi))\right\| \leq C\left(\|\phi\|,\|\psi\|, \cdots,\left\|A^{j} \phi\right\|,\left\|A^{j} \psi\right\|\right)\left\|A^{j} \phi-A^{j} \psi\right\|$

Received by the editors May 6, 1974 .

AMS (MOS) subject classifications (1970). Primary 35L05, 47D05.

Key words and phrases. Nonlinear, wave equation, semigroup. 
for $j=0,1, \cdots, N$ and all $\phi, \psi \in D\left(A^{N}\right)$, where each constant $C$ is a monotone increasing function of the norms indicated. Then, given $\phi_{0} \in D\left(A^{N}\right)$, there is a $T>0$ and a unique $D\left(A^{N}\right)$-valued function on $[0, T)$ which satisfies (1). For each set of the form $\left\{\phi \mid\left\|A^{j} \phi\right\| \leq a_{j}, j=0, \cdots, N\right\}, T$ can be chosen uniformly for all $\phi_{0}$ in the set.

Proof. We will give the proof in the case $N=1$. The proof of the general case is the same but the notation is more cumbersome (see the remark at the end of the proof).

Since $A$ is a closed operator, $D(A)$ is a Banach space under the graph norm $\|\phi\|_{1} \equiv\|\phi\|+\|A \phi\|$. For each $T>0$, let $X_{T}$ denote the set of continuous $D(A)$-valued functions $\phi(\cdot)$ on $[0, T)$ so that

$$
\|\phi(\cdot)\|_{T} \equiv \sup _{t \in[0, T)}\|\phi\|_{1}<\infty .
$$

$X_{T}$ with the norm $\|\cdot\|_{T}$ is a Banach space. Choose some fixed $\epsilon>0$. Let $\phi_{0} \in D(A)$ be given and let $X_{T, \epsilon, \phi_{0}}$ consist of those $\phi(\cdot)$ in $X_{T}$ with $\phi(0)$ $=\phi_{0}$ and $\left\|\phi(\cdot)-\exp (-i A t) \phi_{0}\right\|_{T} \leq \epsilon$. We will show that the map

$$
(s \phi)(t)=\exp (-i A t) \phi_{0}+\int_{0}^{t} \exp (-i A(t-s)) J(\phi(s)) d s
$$

is a contraction on $X_{T, \epsilon, \phi_{0}}$ if $T$ is small enough. We denote by $C_{\epsilon}$ any of the constants in the hypotheses with arguments $\left\|\phi_{0}\right\|+\epsilon$ and $\left\|A \phi_{0}\right\|+\epsilon$. Suppose that $\phi(\cdot) \in X_{T, \epsilon, \phi_{0}}$; then

$$
\begin{array}{r}
\|\exp (-i A(t-(s+b))) J(\phi(s+b))-\exp (i A(t-s)) J(\phi(s))\| \\
\leq\|J(\phi(s+b))-J(\phi(s))\|+\|(\exp (i A b)-I) J(\phi(s))\| \\
\leq C_{\epsilon}\|\phi(s+b)-\phi(s)\|+\|(\exp (-i A b)-I) J(\phi(s))\|
\end{array}
$$

so $\exp (-i A(t-s)) J(\phi(s))$ is a continuous $\mathcal{H}$-valued function of $s$. A similar proof shows that $A \exp (-i A(t-s)) J(\phi(s))$ is also continuous. Therefore, $s \mapsto \exp (-i A(t-s)) J(\phi(s))$ is a continuous $D(A)$-valued function, so

$$
\eta(t) \equiv \int_{0}^{t} \exp (-i A(t-s)) J(\phi(s)) d s
$$

can be defined using the Riemann integral. Thus $\eta(t) \in D(A)$ and

$$
A \int_{0}^{t} \exp (-i A(t-s)) J(\phi(s)) d s=\int_{0}^{t} \exp (-i A(t-s)) A J(\phi(s)) d s
$$

since $A$ is a bounded linear map from $D(A)$ to $\mathcal{H}$ and thus commutes with integration. 
Further,

$$
\begin{aligned}
\|A \eta(t+b)-A \eta(t)\| \leq & \left\|\int_{t}^{t+h} \exp (-i A(t-s)) \exp (-i A b) A J(\phi(s)) d s\right\| \\
& +\left\|\int_{0}^{t}(\exp (-i b A)-I) \exp (i A(t-s)) A J(\phi(s)) d s\right\| \\
\leq & b C_{\epsilon}\|\phi\|_{T}+\int_{0}^{t}\|(\exp (-i b A)-I) A J(\phi(s))\| d s .
\end{aligned}
$$

The integrand in the second term converges to zero as $b \rightarrow 0$ for each $s$ and by the hypotheses on $J$, the integrand is uniformly bounded. Thus, by the dominated convergence theorem, the right-hand side converges to zero as $b \rightarrow 0$, so $A \eta(t)$ is continuous; similarly, $\eta(t)$ is continuous. Further, exactly the same kind of estimates as above show that for any $\phi(\cdot), \psi(\cdot) \in X_{T, \epsilon, \phi}$, we have

$$
\begin{gathered}
\left\|(S \phi)(t)-\exp (-i A t) \phi_{0}\right\| \leq C_{\epsilon} T \sup _{t \in[0, T)}\|\phi(t)\|, \\
\left\|A(S \phi)(t)-A \exp (-i A t) \phi_{0}\right\| \leq C_{\epsilon} T \sup _{t \in[0, T)}\|A \phi(t)\|, \\
\|(S \phi)(t)-(S \psi)(t)\| \leq C_{\epsilon} T \sup _{t \in[0, T)}\|\phi(t)-\psi(t)\|, \\
\|A[(S \phi)(t)-(S \psi)(t)]\| \leq C_{\epsilon} T \sup _{t \in[0, T)}\|A \phi(t)-A \psi(t)\| .
\end{gathered}
$$

Thus, for $T$ small enough, $S$ is a contraction on $X_{T, \epsilon, \phi_{0}}$ and so has a unique fixed point $\phi(\cdot)$ in $X_{T, \epsilon, \phi_{0}}$.

To prove the strong differentiability of $\phi(\cdot)$, we write

$$
\begin{aligned}
\frac{\phi(t+b)-\phi(t)}{b}= & \frac{\exp (-i A h)-I}{b} \exp (-i A t) \phi_{0} \\
& +\frac{1}{b} \int_{t}^{t+h} \exp (-i A(t-s)) \exp (-i b A) J(\phi(s)) d s \\
& +\int_{0}^{t} \exp (-i A(t-s)) \frac{\exp (-i b A)-I}{b} J(\phi(s)) d s
\end{aligned}
$$

Since $\phi_{0} \in D(A)$ the first term converges to $-i A \exp (-i A t) \phi_{0}$ as $b \rightarrow 0$ and since the integrand of the second term is continuous, it converges to $J(\phi(t))$. The integrand of the third term converges to $\exp (-i A(t-s))(-i A J(\phi(s)))$ for each $s$ and

$$
\|((\exp (-i b A)-I) / h) J(\phi(s))\| \leq\|A J(\phi(s))\| \leq C_{\epsilon}\left\|A \phi_{0}\right\|+\epsilon C_{\epsilon}
$$


so the integrand is uniformly bounded. Thus, by the dominated convergence theorem, the third term converges as $b \rightarrow 0$ to $\int_{0}^{t} \exp (-i A(t-s))-i A J(\phi(s)) d s$ which by (3) equals $-i A \int_{0}^{t} \exp (-A(t-s)) J(\phi(s)) d s$. Therefore $\phi(t)$ is strongly differentiable for $t \in[0, T)$ and satisfies (1).

To prove uniqueness, suppose that $\tilde{\phi}(\cdot)$ is a cont inuously differentiable $D(A)$-valued solution of $(1)$ on $[0, \widetilde{T})$. By the differential equation, $A \tilde{\phi}(t)$ is continuous, so $\tilde{\phi} \in X_{T_{0}, \epsilon, \phi_{0}}$ for some $T_{0}>0$. Since $\tilde{\phi}$ obeys $(s \tilde{\phi})(t)=\tilde{\widetilde{\phi}}(t)$ we have $\phi(t)=\tilde{\phi}(t)$ for $0 \leq t<T_{0} \leq T$. Let $T_{1}$ be the sup of such $T_{0}$ and suppose that $T_{1}<T_{0}$. Then $\tilde{\phi}\left(T_{1}\right)=\phi\left(T_{1}\right)$ and by the same argument as above, we conclude that $\tilde{\phi}(t)=\phi(t)$ for $t$ in some small interval $T_{1} \leq t<$ $T_{2}<T$ which contradicts the maximality of $T_{1}$.

The proof for the case $N>1$ is identical except that $X_{T}$ has norm

$$
\|\phi(\cdot)\|_{T}=\sup _{t \in[0, T)} \sum_{j=0}^{N}\left\|A^{j} \phi(t)\right\|
$$

and corresponding changes are made in the definition of $X_{T, \epsilon, \phi_{0}}{ }^{\circ}$

We make several remarks about Theorem 1. First, uniqueness holds in a much stronger sense. Any locally bounded (in $\|\cdot\|$ ) function $\tilde{\phi}(t)$ which satisfies the integral equation $\tilde{\phi}(t)=(S \tilde{\phi})(t)$ is automatically continuous. Thus, by the uniqueness of the contraction mapping fixed point in the larger space of functions $\psi(t)$ which are $\mathcal{H}$-valued, continuous, and close to $\exp (-i A t) \phi_{0}$, we must have $\tilde{\phi}(t)=\phi(t)$.

Secondly, the nonlinear semigroup $\phi_{0} \stackrel{S(t)}{\longrightarrow} \phi(t)$ has the property $S(t)$ : $D\left(A^{j}\right) \rightarrow D\left(A^{j}\right)$ for each $j \leq N$ in analogy with the linear case when $J$ equals zero. This means that the smoothness of the data with respect to. $A$ (which usually is equivalent to smoothness in the space variables) is preserved by $S(t)$.

Finally, notice that we did not need any differentiability assumptions on $J$ to get strong solutions to equation (1). The strong differentiability followed automatically from the estimate $\left(\mathrm{H}_{1}\right)$ and the fact that $J$ occurs under the integral in equation (2). To get higher differentiability in $t$ we must make differentiability assumptions on $J$.

Theorem 2. Suppose that in addition to the hypotheses of Theorem 1, J satisfies the following property: If $\phi(t)$ is a $j$ times strongly differentiable $D\left(A^{N}\right)$-valued function so that $\phi^{(k)}(t) \in D\left(A^{N-k}\right)$ and $A^{N-k} \phi^{(k)}(t)$ is continuous for all $k \leq j$, then $J(\phi(t))$ is $j$ times strongly differentiable, $(d / d t)^{j} J(\phi(t)) \in D\left(A^{N-j-1}\right)$ and $A^{N-j-1}(d / d t)^{j} J(\phi(t))$ is continuous. If $J$ 
satisfies this property then the solution of Theorem 1 is $N$ times strongly continuously differentiable and $\phi^{(j)}(t) \in D\left(A^{N-j}\right)$ for all $j \leq N$.

Proof. The proof is by induction. We know that the solution $\phi(t)$ is strongly continuously differentiable and satisfies equation (1). Further, by (3),

$$
A \phi(t)=\exp (-i A t) A \phi_{0}+\int_{0}^{t} \exp (-i A(t-s)) A J(\phi(s)) d s
$$

so the same argument as in Theorem 1 shows that $A \phi(t)$ is continuously differentiable and

$$
d(-i A \phi(t)) / d t=(-i A)^{2} \phi(t)-i A J(\phi(t)) .
$$

By the hypothesis on $J, J(\phi(t))$ is continuously differentiable so since (1) holds, $\phi(t)$ is twice continuously differentiable and

$$
\phi^{\prime \prime}(t)=(-i A)^{2} \phi(t)-i A J(\phi(t))+d J(\phi(t)) / d t .
$$

We now know that $\phi(t)$ is twice strongly differentiable and that

$$
A^{N-2} d J(\phi(t)) / d t
$$

is strongly continuous so we can use the hypothesis on $J$ again to conclude that $\phi(t)$ is three times strongly differentiable and so forth.

In general, global solutions in $t$ will not exist [3]. However, in some cases an energy inequality enables one to find a priori bounds on the growth of $\|\phi(t)\|$. This combined with slightly stronger estimates is sufficient to guarantee strong global smooth solutions.

Theorem 3. Let $N$ be fixed and suppose that $A$ and $J$ satisfy the hypotheses of Theorem 1 except that $\left(\mathrm{H}_{j}\right)$ for $1 \leq j \leq N$ is replaced by

$$
\left\|A^{j} J(\phi)\right\| \leq C\left(\|\phi\|, \cdots,\left\|A^{j-1} \phi\right\|\right)\left\|A^{j} \phi\right\| \text {. }
$$

That is, the constant does not depend on $\left\|A^{j} \phi\right\|$. Suppose that on any finite interval $[0, T)$ where the strong solution $\phi(t)$ of $(1)$ exists, $\|\phi(t)\|$ is bounded. Then the strong solution exists globally in $t$ and $\phi(t) \in D\left(A^{N}\right)$ for all $t$.

Proof. Let $\left[0, T_{0}\right)$ be the maximal interval on which a strongly differentiable solution of (1) with values in $D\left(A^{N}\right)$ exists. If $T_{0}<\infty$ then $\sum_{j=0}^{N}\left\|A^{j} \phi(t)\right\|$ must go to infinity as $t \uparrow T_{0}$. Otherwise, we could continue the solution across $T_{0}$ by using the fact that the $T$ in Theorem 1 can be chosen uniformly for $\left\|A^{j} \phi_{0}\right\|$ in bounded sets. Thus, to conclude global existence we need only show that $\sum_{j=0}^{N}\left\|A^{j} \phi(t)\right\|$ is bounded on any finite interval $[0, T)$ where the solution exists. 
By hypothesis $\|\phi(t)\|$ is bounded on $[0, T)$. Thus,

$$
C_{1}=\sup _{t \in[0, T)} C(\|\phi(t)\|)<\infty,
$$

so since $\phi$ satisfies $\phi(t)=(S \phi)(t)$ we have

$$
\|A \phi(t)\| \leq\left\|A \phi_{0}\right\|+\int_{0}^{t}\|A J(\phi(s))\| d s \leq\left\|A \phi_{0}\right\|+C_{1} \int_{0}^{t}\|A \phi(s)\| d s .
$$

Thus, by iteration, $\|A \phi(t)\| \leq\left\|A \phi_{0}\right\| \exp \left(C_{1} t\right)$ so $\|A \phi(t)\|$ is bounded on $[0, T)$. Similarly,

$$
\left\|A^{2} \phi(t)\right\| \leq\left\|A^{2} \phi_{0}\right\|+C_{2} \int_{0}^{t}\left\|A^{2} \phi(s)\right\| d s
$$

where $C_{2}=\sup _{t \in[0, T]} C(\|\phi(t)\|,\|A \phi(t)\|)$. Since $\|\phi(t)\|$ and $\|A \phi(t)\|$ are bounded on $[0, T)$, so $C_{2}<\infty$, which implies by iteration that $\left\|A^{2} \phi(t)\right\|$ is bounded on $[0, T)$. Continuing in this way we show that $\left\|A^{j} \phi(t)\right\|$ is bounded on $[0, T)$ for each $j \leq N$.

The following theorem follows immediately from the previous three. In applications it is used to show that if the data are $C^{\infty}$ then the solution is $C^{\infty}$.

Theorem. Let $A$ and $J$ satisfy the hypotheses $\left(\mathrm{H}_{j}^{\prime}\right)$ and $\left(\mathrm{H}_{j}^{L}\right)$ for all $j$. Suppose that $J$ satisfies the hypothesis in Theorem 2 for all $N$ and that any strong solution of (1) is a priori bounded on any finite interval. If $\phi_{0} \epsilon$ $\bigcap_{j=1}^{\infty} D\left(A^{j}\right)$, then the solution $\phi(t)$ of $(1)$ is infinitely often strongly differentiable in $t$ and each derivative $t$ has values in $\bigcap_{j=1}^{\infty} D\left(A^{j}\right)$.

In applications, the estimates $\left(\mathrm{H}_{j}^{\prime}\right)$ and $\left(\mathrm{H}_{j}^{L}\right)$ are typically proven by Sobolev estimates. For example, for the Klein-Gordon equation $\square u+m^{2} u=-u^{3}$ in $\mathbf{R}^{3}$ one only needs the estimate $\|u\|_{6} \leq C\left\|\sqrt{-\Delta+m^{2}} u\right\|_{2}$. For details, see Segal [6], Chadam [1], or Reed and Simon [5].,

We can now explain the relationship of these theorems to Segal's methods and results. Segal first proves the existence of solutions of $\phi(t)=(S \phi)(t)$ under the condition that $J$ be Lipschitz. He then investigates smoothness as a separate question and proves essentially that if $J$ has the properties of Theorems 1 and 2 without the estimates, then the conclusions hold. For example, a typical hypothesis would be $J: D\left(A^{j}\right) \rightarrow D\left(A^{j}\right)$ but there is no assumed estimate on $\left\|A^{j} J(\phi)\right\|$ in terms of $\left\|A^{k} \phi\right\|, k=0,1, \cdots, j$. Segal's theorem is very nice since it shows that the general property of being in the domain of powers of a closed operator is already strong enough to guarantee the invariance of the domain under the nonlinear semigroup. However, in prac- 
tice, the only way one can prove facts like $J: D\left(A^{j}\right) \rightarrow D\left(A^{j}\right)$ is by using estimates like those above. And, in the presence of these estimates, the theorems can be proven by the elementary methods we have indicated.

\section{BIBLIOGR APHY}

1. J. Chadam, Asymptotics for $\square u=m^{2}+G\left(x, t, u, u_{x}, u_{t}\right)$. I and II: Global existence and decay, Ann. Scuola Norm. Sup. Pisa 26 (1972), 33-65, 67-95.

2. - Global solutions of the Cauchy problem for the (classical) coupled Maxwell-Dirac equations in one space dimension, J. Functional Analysis 13 (1973), 173-184.

3. J. B. Keller, On solutions of nonlinear wave equations, Comm. Pure Appl. Math. 10 (1957), 523-530. MR 20 \#3371.

4. W. Littman, The wave operator and $L^{p}$ norms, J. Math. Mech. 12 (1963), 55-68. MR 26\#4043.

5. M. Reed and B. Simon, Methods of modern mathematical physics, Vol. II: Fourier analysis, self-adjointness, Academic Press, New York, 1975. $27 \# 2879$.

6. I. Segal, Non-linear semi-groups, Ann. of Math. (2) 78 (1963), 339-364. MR

7. W. Strauss, The energy method in nonlinear partial differential equations, Math. Notes, no. 47, Inst. Mat. Pura Appl., Rio de Janeiro, 1969. MR 42 \#8051.

DEPARTMENT OF MATHEMATICS, PRINCETON UNIVERSITY, PRINCETON, NEW JERSEY 08540

Current address: Department of Mathematics, Duke University, Durham, North Carolina 27701 